\title{
Extension of the nuclear reaction model code EMPIRE to actinides' nuclear data evaluation
}

\author{
R. Capote ${ }^{1, a}$, M. $\operatorname{Sin}^{2}$, A. Trkov ${ }^{3}$, M. Herman ${ }^{4}$, B.V. Carlson ${ }^{5}$, and P. Obložinský ${ }^{4}$ \\ 1 Nuclear Data Section, International Atomic Energy Agency, 1400 Vienna, Austria \\ 2 University of Bucharest, Bucharest, Romania \\ 3 Jozef Stefan Institute, Ljubljana, Slovenia \\ ${ }^{4}$ National Nuclear Data Center, Brookhaven National Laboratory, Upton, NY, USA \\ 5 Instituto Tecnológico de Aeronáutica, São José dos Campos SP, Brazil
}

\begin{abstract}
Recent extensions and improvements of the EMPIRE code system are outlined. They add new capabilities to the code, such as prompt fission neutron spectra calculations using Hauser-Feshbach plus pre-equilibrium prefission spectra, cross section covariance matrix calculations by Monte Carlo method, fitting of optical model parameters, extended set of optical model potentials including new dispersive coupled channel potentials, paritydependent level densities and transmission through numerically defined fission barriers. These features, along with improved and validated ENDF formatting, exclusive/inclusive spectra, and recoils make the current EMPIRE release a complete and well validated tool for evaluation of nuclear data at incident energies above the resonance region. The current EMPIRE release has been used in evaluations of neutron induced reaction files for ${ }^{232} \mathrm{Th}$ and ${ }^{231,233} \mathrm{~Pa}$ nuclei in the fast neutron region at IAEA. Triple-humped fission barriers and exclusive pre-fission neutron spectra were considered for the fission data evaluation. Total, fission, capture and neutron emission cross section, average resonance parameters and angular distributions of neutron scattering are in excellent agreement with the available experimental data.
\end{abstract}

\section{Introduction}

EMPIRE belongs to a new generation of nuclear reaction codes, and is intended as a general theoretical tool to be used both in basic research and nuclear data evaluation over a broad range of incident energies and projectiles. It is designed to contain up-to-date nuclear reaction models as well as to be easy to use. The currently available version 2.19 of the EMPIRE (Lodi) [1,2] was released in March 2005. A comprehensive library of input parameters based mostly on the RIPL-2 library [3] and a graphical user interface facilitate preparation of the input and user-friendly operation of the code. A large part of the system is dedicated to ENDF formatting, allowing the generation of ENDF-6 formatted and validated files, which are ready for further processing with the NJOY code.

Over the two years since the release of version 2.19 , the EMPIRE system has been continuously extended, validated and improved to meet evaluation exigencies posed by the production of modern data libraries. In particular, the development of the ENDF/B-VII.0 library [4] was a unique occasion for a first large scale deployment of the EMPIRE code as described in another contribution [5]. EMPIRE has been used for 77 of the 393 neutron evaluations contained in the ENDF/B-VII.0 library [4], including the evaluations of the ${ }^{232} \mathrm{Th}$ and ${ }^{231,233} \mathrm{P}$ nuclei which were undertaken within an IAEA CRP [6] and later adopted for the ENDF/B-VII.0 library.

The international team of developers added a number of new features. In particular, automatic fitting of optical potential parameters, improvements in ENDF-6 formatting,

\footnotetext{
${ }^{a}$ Presenting author, e-mail: r.capotenoy@iaea.org
}

treatment of the resonance region and plotting and generation of cross section covariances in the fast neutron energy range by a EMPIRE/KALMAN sequence were added as described in other contributions to this conference $[5,7]$. Below we will give a summary of recent changes and elaborate more on the use of the EMPIRE system for the evaluation of neutron induced reactions on actinide nuclei in the fast neutron energy range. In fact, the evaluations for ${ }^{232} \mathrm{Th}$ and ${ }^{231,233} \mathrm{P}$ nuclei were the only ones involving fissile nuclei produced by the EMPIRE system and adopted for the ENDF/B-VII.0 release.

\section{EMPIRE extensions and improvements}

Let us review some of the recently introduced changes:

Full support for dispersive coupled-channel optical model (DCCOM) potentials has been introduced. In particular, the DCCOM potential [8] derived for the ${ }^{232}$ Th nucleus provided an excellent description of the total cross-section database available for thorium from $1 \mathrm{keV}$ up to $200 \mathrm{MeV}$ as can be seen in figure 1, and laid solid ground for the good description achieved in other neutron channels, in particular for the total inelastic and ${ }^{232} \mathrm{Th}(\mathrm{n}, 2 \mathrm{n})$ reactions as shown in figure 2 . The good agreement reached with the available total inelastic data is especially remarkable; given the on-going discussions of the important role played by these data in the energy region around $1.5-3 \mathrm{MeV}$ where the neutron inelastic cross-section reaches its maximum. This EMPIRE extension will allow one to use the recently derived DCCOM potential for the actinide region $[9,10]$ in future evaluations.

DWBA calculations on odd targets have been incorporated to enable use of a direct mechanism when a coupled-channel 


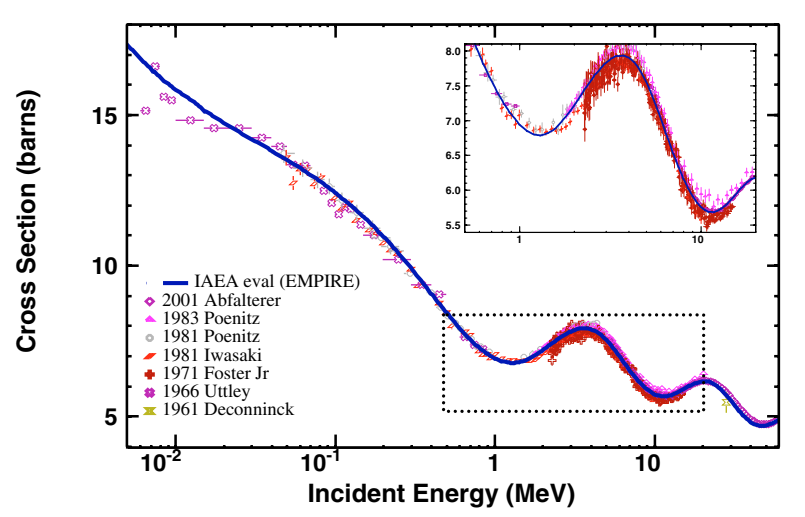

Fig. 1. Evaluated total cross section for neutrons incident on ${ }^{232} \mathrm{Th}$ (DCCOM [8]). The dotted region is expanded in the inset.

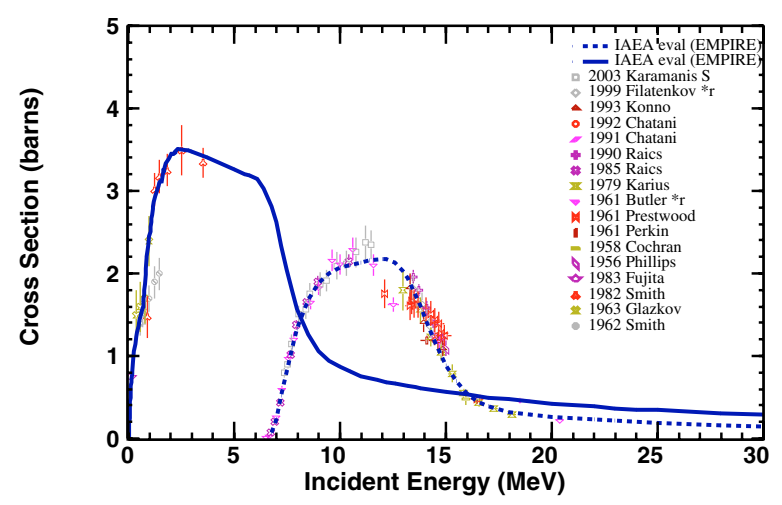

Fig. 2. Total inelastic and $(n, 2 n)$ cross-sections of ${ }^{232} \mathrm{Th}$. The dashed blue line corresponds to the $(n, 2 n)$ reaction.

(CC) potential is not available or it is necessary to use a direct reaction mechanism for levels that can not be included within the CC coupling scheme. Since DWBA calculations can not be performed on targets with non-integer spins, actual computations are done for a neighbouring nucleus with an even number of nucleons. This enhancement was crucial for a successful evaluation of the neutron induced reactions on odd protactinium isotopes by using the well characterized collective structure of the ${ }^{232} \mathrm{Th}$ nucleus.

DWBA calculations to discrete levels embedded in the continuum have been included as a means of increasing the high energy part of the neutron spectra It compensates for shortcomings of the reaction models that in certain cases are unable to properly account for collective states at high excitation energies. The new model successfully reproduced spectra of emitted neutrons on ${ }^{232} \mathrm{Th}$ in the whole energy range of interest as can be seen in figure 3 .

Monte Carlo sampling calculation of the covariance matrix of model parameters and subsequent statistical processing of calculated cross-sections has been added.

In 2004, D.L Smith [11] suggested a new approach to nuclear data evaluation that incorporates Monte Carlo simulation. The method has been successfully implemented in the EMPIRE code. It was employed to generate a comprehensive neutron reaction evaluation including covariances for the

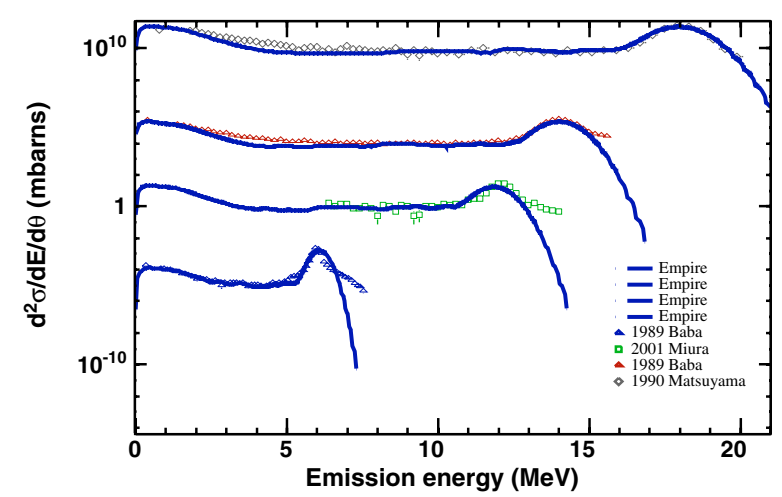

Fig. 3. Neutron emission cross sections at 30 degrees for $6.1,11.9$, 14.1 and $18 \mathrm{MeV}$ incident neutron energy on ${ }^{232} \mathrm{Th}$ (scaled by $1,10^{3}$, $10^{6}$ and $10^{9}$ respectively). Fission neutrons were not included.

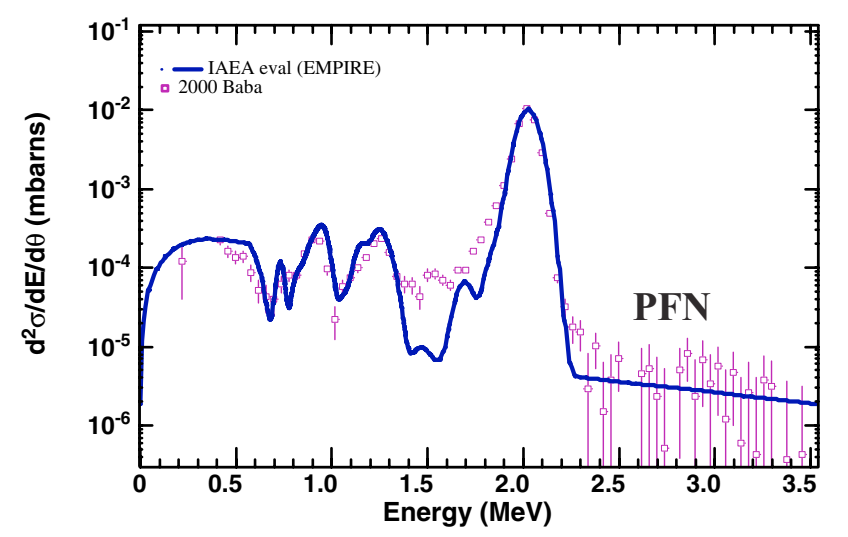

Fig. 4. Neutron emission cross sections at 30 degrees for $2.03 \mathrm{MeV}$ incident neutron energy on ${ }^{232} \mathrm{Th}$. The fission neutron contribution is shown above the elastic peak located at $\sim 2 \mathrm{MeV}$.

IAEA ${ }^{232}$ Th evaluation adopted for the ENDF/B-VII.0 library [12]. The merging of experimental and modelling results was performed in this evaluation by using a generalized-leastsquares method, as implemented in the GANDR system [13].

Prompt fission neutron (PFN) spectra and average number of prompt fission neutron $(\bar{v})$ calculations are important additions that make the evaluated files for actinides complete. Furthermore, both $\bar{v}$ and PFN spectra are also consistent with the calculated fission cross sections. The calculated PFN spectra include both post-fission neutrons emitted from fully accelerated fragments and a properly weighted contribution of pre-fission neutrons [14]. A sudden drop of the PFN average energy above the emissive fission threshold predicted by Maslov [14] is naturally obtained in our calculations. This behaviour can not be reproduced by the Los Alamos PFN model without considering pre-equilibrium neutron emission. A typical PFN contribution to the neutron emission spectra at $2 \mathrm{MeV}$ of incident energy is shown in figure 4.

Improvement of the fission channel has been continuous. An already sophisticated fission model [2] based on the optical model for fission developed to describe triple-humped barrier in light actinides [15] has been further refined [16]. Current advances include absorption in the third well and the capability to use numerical fission barriers [17] and level densities 


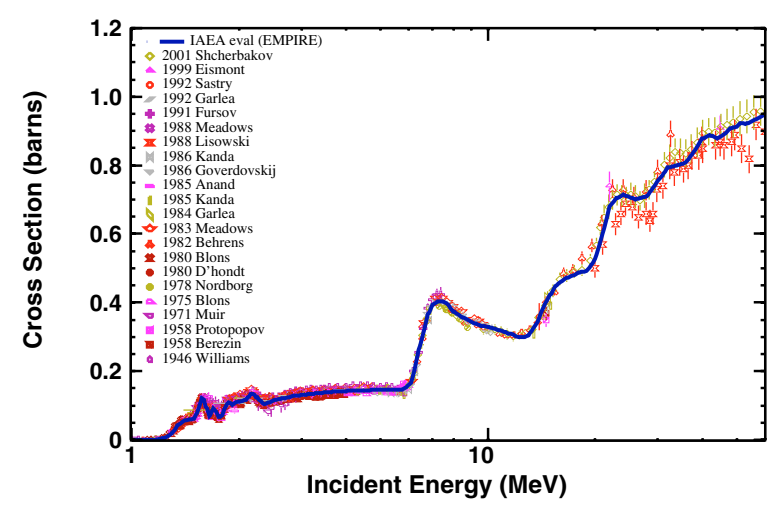

Fig. 5. Neutron-induced fission cross section of ${ }^{232} \mathrm{Th}$ up to $60 \mathrm{MeV}$. Experimental data taken from EXFOR as quoted.

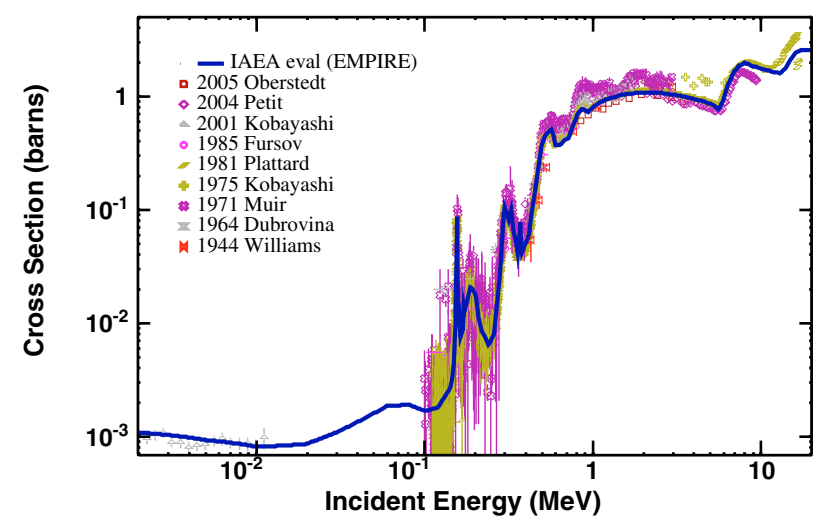

Fig. 6. Neutron-induced fission cross section of ${ }^{231} \mathrm{~Pa}$.

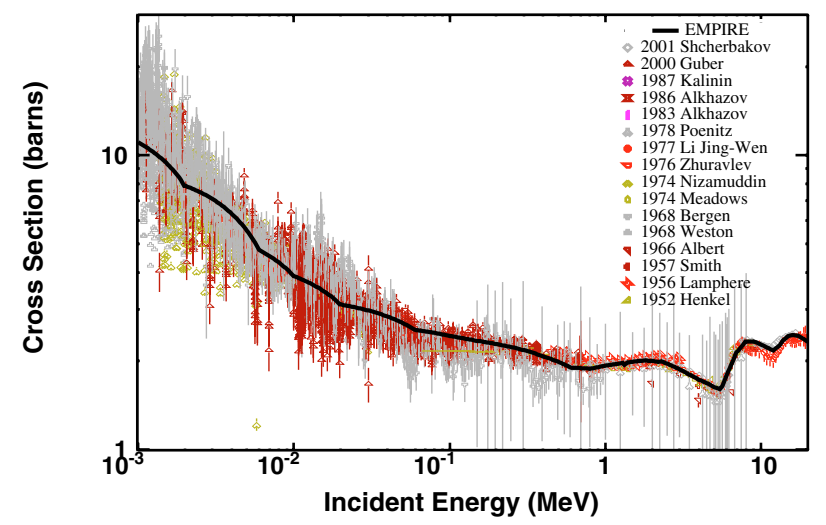

Fig. 7. Neutron-induced fission cross section of ${ }^{233} \mathrm{U}$.

$[18,19]$, such as those obtained from microscopic HartreeFock-Bogolyubov calculations, for fission cross-section calculations as shown in ref. [16]. The quality achieved in the description of the neutron induced fission cross-section can be judged in figures 5-7 showing the EMPIRE calculations of the ${ }^{232} \mathrm{Th}(\mathrm{n}, \mathrm{f}),{ }^{231} \mathrm{~Pa}(\mathrm{n}, \mathrm{f})$ and ${ }^{233} \mathrm{U}(\mathrm{n}, \mathrm{f})$ reactions. It should be mentioned that ${ }^{233} \mathrm{U}$ results shown in the figure 7 are very preliminary, while the other two are final evaluations.

It is also noteworthy that the EMPIRE built-in fission model provides descriptive capabilities. An attempt to evaluate our current predictive capabilities in neutron-induced fission cross-section calculations is presented in ref. [16]. The

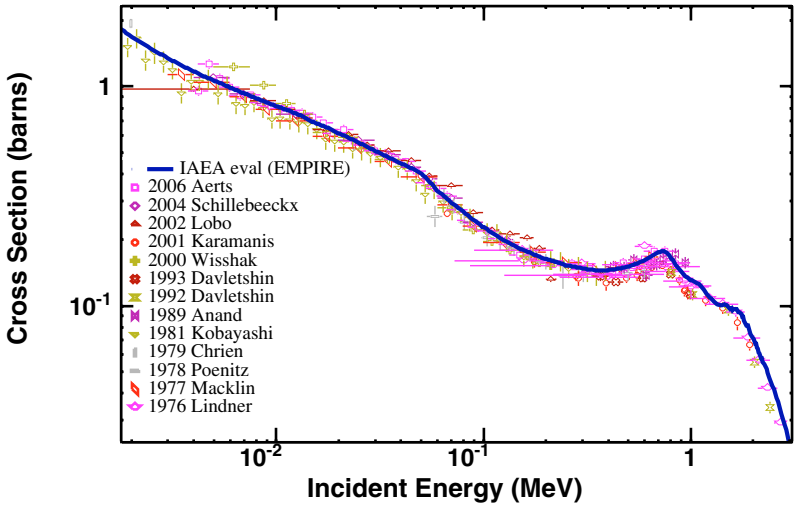

Fig. 8. Neutron capture cross section of ${ }^{232} \mathrm{Th}$.

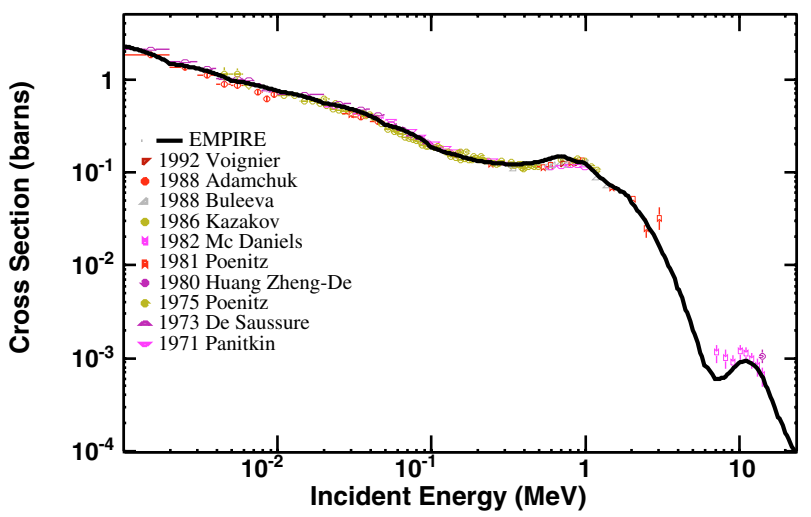

Fig. 9. Neutron capture cross section of ${ }^{238} \mathrm{U}$.

descriptive capabilities of the EMPIRE fission calculation are quite important for actinide evaluations as they provide a needed constraint for the consistent description of competing neutron emitting reactions.

The modified Lorentzian (MLO) gamma ray strength function developed by Plujko [2] was implemented in the previous EMPIRE release [1]. The implementation has been extensively validated in recent actinides' evaluations allowing for an excellent reproduction of the available high-quality capture cross section database as can be seen in figures 8 and 9 showing the EMPIRE calculated neutron capture on ${ }^{232} \mathrm{Th}$ and ${ }^{238} \mathrm{U}$, respectively.

\section{Conclusions}

Several important extensions and improvements of the physical model core of the EMPIRE system have been introduced over the last two years. EMPIRE has been additionally validated by its successful use in recent evaluations of neutroninduced reactions on actinide nuclei. We have seen that EMPIRE calculations in the fast neutron region are able to reproduce the available experimental data for total, elastic and inelastic neutron scattering, neutron capture, neutron emission and the neutron-induced fission cross section of major actinide nuclei. We are confident that EMPIRE system is ready to be used in future physical studies and evaluations of fissioning nuclei. 


\section{References}

1. M. Herman, P. Obložinský, R. Capote, M. Sin, A. Trkov, A. Ventura, V. Zerkin, in Proceedings of the International Conference on Nuclear Data for Science and Technology, 27 Sept.-1 Oct. 2004, Santa Fé, NM, USA.; AIP Conf. Proc. 769, 1184 (2005). Online at www.nndc.bnl.gov/empire219/.

2. M. Sin, R. Capote, M. Herman, P. Obložinský, A. Ventura, A. Trkov, in Proceedings of the International Conference on Nuclear Data for Science and Technology, 27 Sept.-1 Oct. 2004, Santa Fe, NM, USA.; AIP Conf. Proc. 769, 1249 (2005).

3. T. Belgya, O. Bersillon, R. Capote et al., Handbook for calculations of nuclear reaction data: Reference Input Parameter Library-2, Technical Report IAEA-TECDOC-1506 (IAEA, Vienna, Austria, 2006). Available online @ http://wwwnds.iaea.org/RIPL-2/.

4. M.B. Chadwick et al., Nucl. Data Sheets 107, 2931 (2006) .

5. M. Herman et al. (these proceedings).

6. A. Trkov, A IAEA Coordinated Research Project on Evaluated nuclear data for thorium-uranium fuel cycle. Information available online at www-nds.iaea.org/Th-U/.

7. D. Rochman et al. (these proceedings)

8. E.Sh. Soukhovitskii, R. Capote, J.M. Quesada, S. Chiba, Phys. Rev. C 72, 024604 (2005).
9. R. Capote, E.Sh. Soukhovitskii, J.M. Quesada, S. Chiba, Phys. Rev. C 72, 064610 (2005).

10. R. Capote, E.Sh. Soukhovitskii, J.M. Quesada, S. Chiba (these proceedings).

11. D.L. Smith, Covariance Matrices for Nuclear Cross-Sections Derived from Nuclear Model Calculations, Technical report ANL/NDM-159 (Argonne National Laboratory, USA, 2005).

12. A. Trkov, R. Capote, Cross-Section Covariance Data, Documentation of the Th-232 Evaluation for ENDF/B-VII.O (MAT $=9040 M F=1 M T=451)$, National Nuclear Data Center, Brookhaven National Laboratory (http://www.nndc.bnl.gov), 15 December 2006.

13. D.W. Muir, Global Assessment of Nuclear Data Requirements - the GANDR Project, an IAEA Nuclear Data Section Data Development Project. Available online at http://wwwnds.iaea.org/gandr/.

14. V.M. Maslov, Eur. Phys. J. A 18, 93 (2003).

15. M. Sin, R. Capote, A. Ventura et al., Phys. Rev. C 74, 014608 (2006).

16. M. Sin, R. Capote, S. Hilaire, S.Goriely, A. Koning (these proceedings).

17. S. Goriely, J.M. Pearson (these proceedings).

18. S. Hilaire, S.Goriely, Nucl. Phys. A 779, 63 (2006).

19. S. Hilaire, S.Goriely (these proceedings). 